Supporting Information

\title{
Visible Color Tunable Emission in Three-dimensional Light Emitting Diodes by MgO Passivation of Pyramid Tip
}

Ji-Hyun Kim ${ }^{\dagger}$, Byeong Uk Ye ${ }^{\dagger}$, Joonmo Park ${ }^{\dagger}$, Chul Jong Yoo ${ }^{\ddagger}$, Buem Joon Kim ${ }^{\ddagger}$, Hu Young Jeong $^{\S}$, Jin-Hoe Hur", Jong Kyu Kim", Jong-Lam Lee ${ }^{\ddagger}$ and Jeong Min Baik ${ }^{\dagger *}$

${ }^{\dagger}$ Department of Materials Science Engineering, KIST-UNIST-Ulsan Center for Convergent Materials, Ulsan National Institute of Science and Technology (UNIST), Ulsan 689798,Republic of Korea.

${ }^{\ddagger}$ Department of Materials Science and Engineering, Division of Advanced Materials Science, Pohang University of Science and Technology (POSTECH), Pohang, Gyeongbuk 790-784, Republic of Korea.

${ }^{\S}$ UNIST Central Research Facilities (UCRF), Ulsan National Institute of Science and Technology (UNIST), Ulsan, Korea

"UNIST-OLYMPUS BIOMED IMAGING CENTER(UOBC), Ulsan National Institute of Science and Technology (UNIST), Ulsan, Republic of Korea

" Department of Materials Science and Engineering, Pohang University of Science and Technology (POSTECH), Pohang, Gyeongbuk 790-784, Republic of Korea.

* Corresponding author : jbaik@unist.ac.kr 
(a)
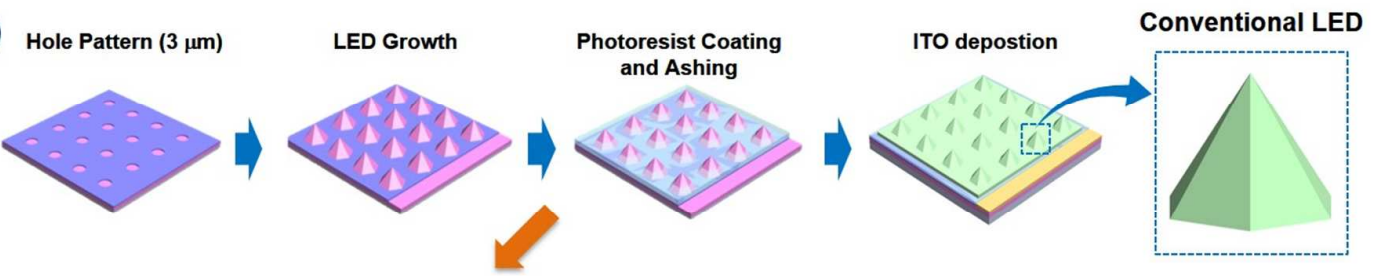

(b)
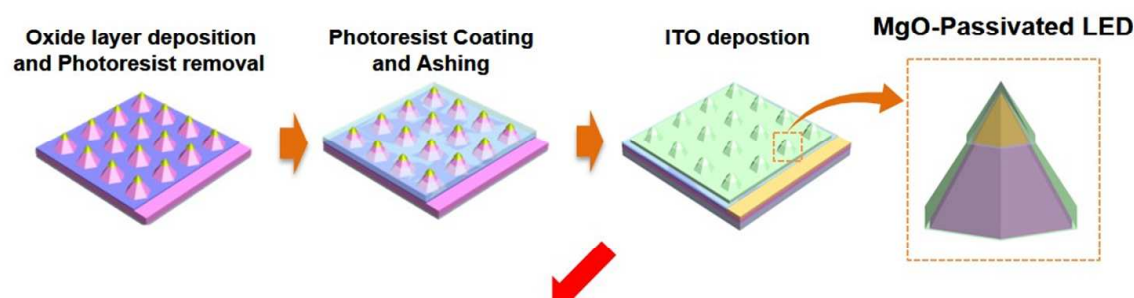

(c)

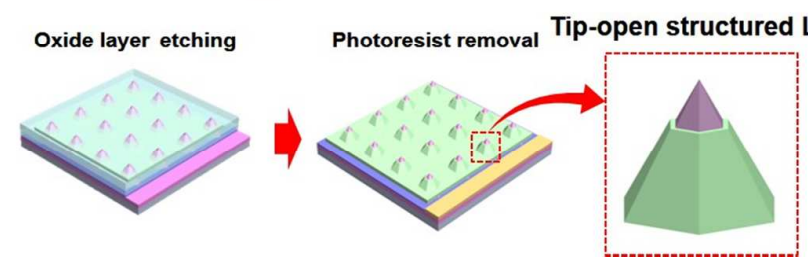

Figure S1. The fabrication schematic process of (a) Conventional LEDs, (b) MgO-passivated LEDs and (c) Tip-open structured LEDs

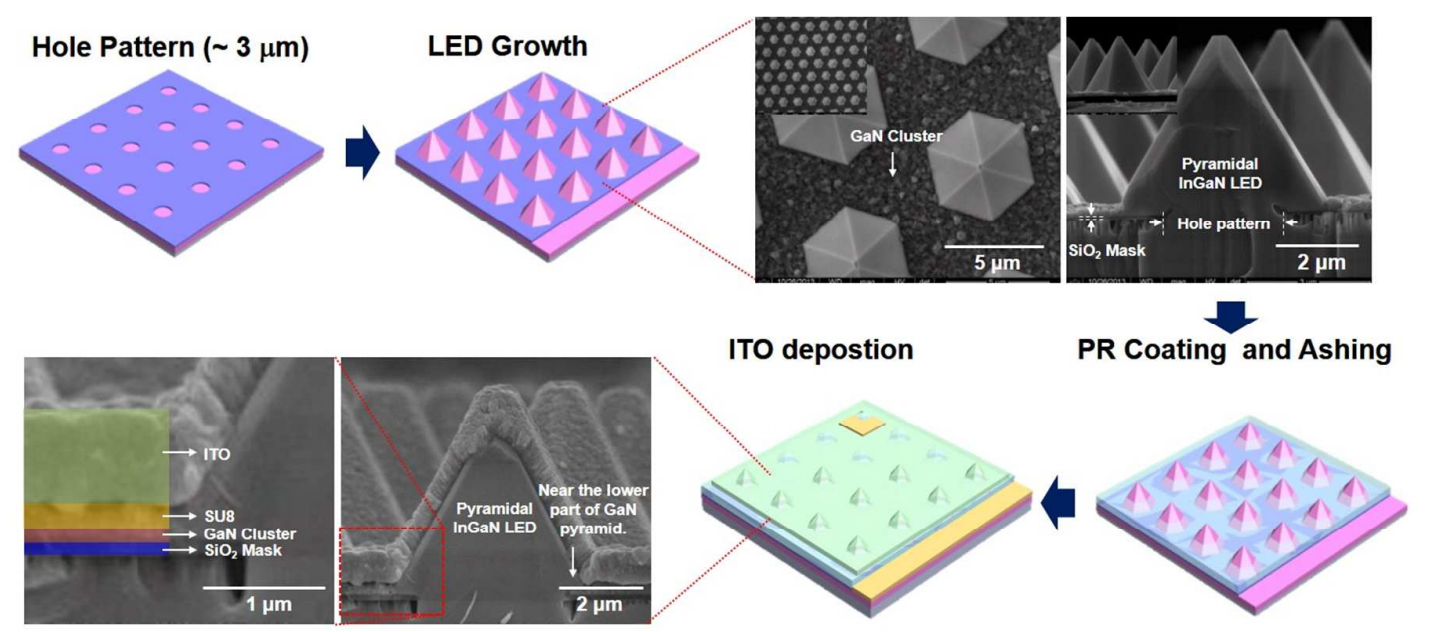

Figure S2. The fabrication schematic process and SEM images of Conventional LEDs. 

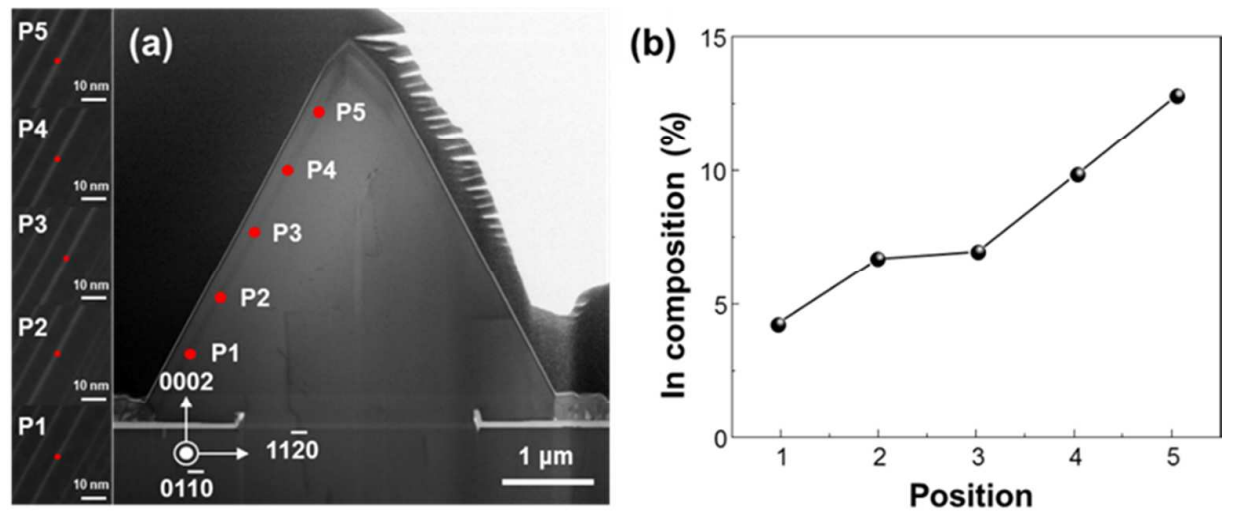

Figure S3. The cross-sectional TEM images of a LED. (a) Cross-sectional low-magnification image of single LED covered by MQWs. (b) In composition plot from the pyramid InGaN MQWs at various positions. The measured points are marked in (a)

(a)

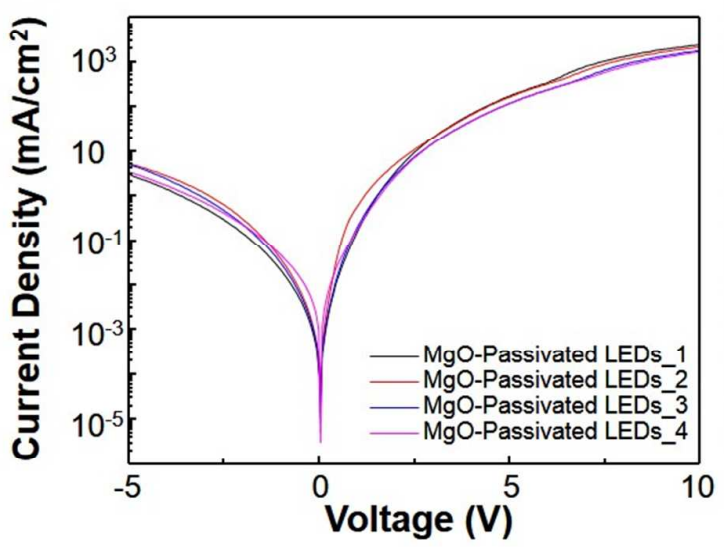

(b)

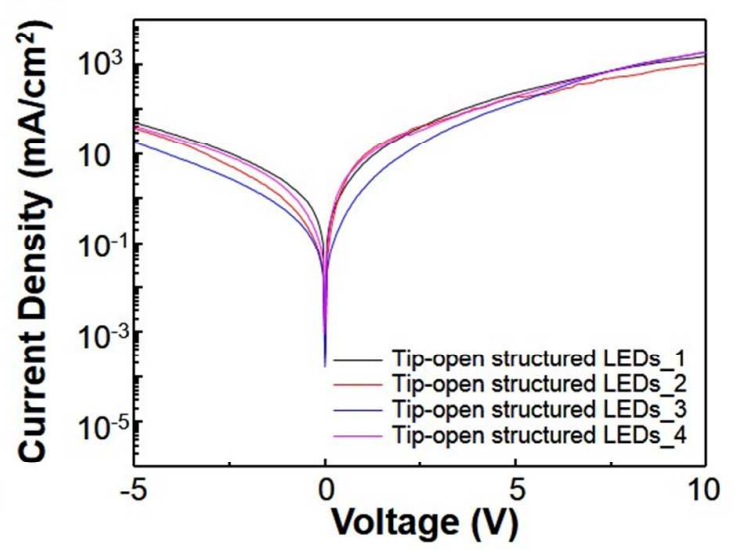

Figure S4. Current-voltage curves measurements from different samples for (a) MgOpassivated LED and (b) Tip-open structured LED 